\title{
An Unusual Case of Gastritis in One Patient Receiving PD-1 Blocking Therapy: Coexisting Immune-Related Gastritis and Cytomegaloviral Infection
}

\author{
Jun Lu ${ }^{\mathrm{a}}$, Roberto J. Firpi-Morell ${ }^{\mathrm{b}}$, Long H. Dang ${ }^{\mathrm{c}}$, Jinping Lai ${ }^{\mathrm{d}}$, Xiuli Liu ${ }^{\mathrm{d}}$ e
}

\begin{abstract}
The programmed death 1 (PD-1), programmed death ligand 1 (PD-L1) and cytotoxic T lymphocyte-associated antigen 4 (CTLA-4) immune checkpoints are negative regulators of T-cell immune function. Inhibition of these targets by antibodies (PD-1 blocking therapy) has been explored to treat solid malignancies such as melanoma, non-small cell lung cancer and other cancers. PD-1 blocking therapy is known to cause gastrointestinal tract adverse events in some patients and some of the adverse events are thought to be immune-mediated. Cancer patients receiving PD-1 blocking therapy have often failed several lines of chemotherapy and thus potentially are susceptible to a variety of infections including cytomegaloviral infection. However, there has not been any report of concurrent immune-mediated gastroenterocolitis and cytomegaloviral infection in cancer patients receiving PD-1 blocking therapy. Herein, we report one unusual case of histologically confirmed gastritis with features of immune-mediated pangastritis and cytomegaloviral infection in one patient who had metastatic urothelial carcinoma and received PD-1 blocking therapy, initially with atezolizumab (anti-PD-L1 antibody) followed by a switch to pembrolizumab (anti-PD-1 antibody) because of tumor progression. Pembrolizumab was held and intravenous ganciclovir treatment was started, the patient's symptoms (abdominal pain and vomiting) were significantly improved and she was discharged from the hospital in stable conditions on hospital day 5. Pathologists should be aware of PD-1 blocking therapy-associated immune-mediated gastrointestinal tract adverse effect and concurrent cytomegaloviral infection.
\end{abstract}

Keywords: Programmed death 1; Programmed death ligand 1; PD-1 blocking therapy; Gastritis; Cytomegalovirus

Manuscript submitted July 4, 2018, accepted July 30, 2018

aDepartment of Pathology, Beijing Chaoyang Hospital, Capital University, Beijing, China

${ }^{b}$ Department of Gastroenterology, Hepatology, and Nutrition, University of Florida, Gainesville, FL, USA

'Department of Medicine, University of Florida, Gainesville, FL, USA

dDepartment of Pathology, Immunology, and Laboratory Medicine, University of Florida, Gainesville, FL, USA

${ }^{e}$ Corresponding Author: Xiuli Liu, Department of Pathology, Immunology, and Laboratory Medicine, University of Florida, PO Box 100275, Gainesville, FL 32610, USA. Email: xiuliliu@ufl.edu

doi: https://doi.org/10.14740/gr1068w

\section{Introduction}

The programmed death 1 (PD-1), programmed death ligand 1 (PD-L1) and cytotoxic T lymphocyte-associated antigen 4 (CTLA-4) immune checkpoints are negative regulators of Tcell immune function [1]. Inhibition of these targets by antibodies, pembrolizumab (KEYTRUDA, anti-PD-1), nivolumab (OPDIVO, anti-PD-1), atezolizumab (Tecentriq, anti-PD-L1) and ipilimumab (antibody against CTLA-4), resulting in increased activation of the immune system, has been explored to treat melanoma, non-small cell lung cancer and other cancers [1-3]. Both pembrolizumab and nivolumab are approved for treatment of metastatic melanoma, nivolumab also for squamous non-small cell lung cancer after prior chemotherapy. Atezolizumab (Tecentriq), a fully humanized, engineered monoclonal antibody of $\operatorname{IgG} 1$ isotype against the protein PD-L1, has been used as an immunotherapy for several types of solid tumors including metastatic non-small cell lung cancer and locally advanced or metastatic bladder urothelial carcinoma that has progressed after platinum-containing chemotherapy. Gastrointestinal adverse effects are common after a treatment with checkpoint inhibitors and include diarrhea, decreased appetite, nausea, pyrexia and dyspepsia, and some of these adverse events may be immune-mediated [3-8]. Most patients with PD-1 blockade-associated gastrointestinal tract injury presented with diarrhea and the inflammatory process usually involved multiple sites of the gastrointestinal tract. Rare case of PD-1 blockage-associated adverse event may only affect the stomach. For example, in one series, one patient $(5 \%)$ presented with only nausea, vomiting, volume depletion and fatigue and was found to have congested, erythematous and granular gastric mucosa on endoscopy [9]. Histological findings in the gastric mucosa from patients receiving anti-PD 1 antibodies who had gastrointestinal symptoms are lymphoplasmacytic expansion of the lamina propria and intra-epithelial neutrophils, gland abscesses, crypt apoptosis and lamina propria eosinophils [9].

Cytomegaloviral gastritis is a rare disease, even in immunocompromised patients. Characteristic findings on upper endoscopy are punched-out gastric ulcers. Rare cases of cytomegaloviral gastritis can present as hemorrhagic, necrotic gastritis with inflammatory bridging [10]. In rare cases, it may present as a mass lesion, mimicking lymphoma [11]. In pediatric population, cytomegaloviral infection is associated with 


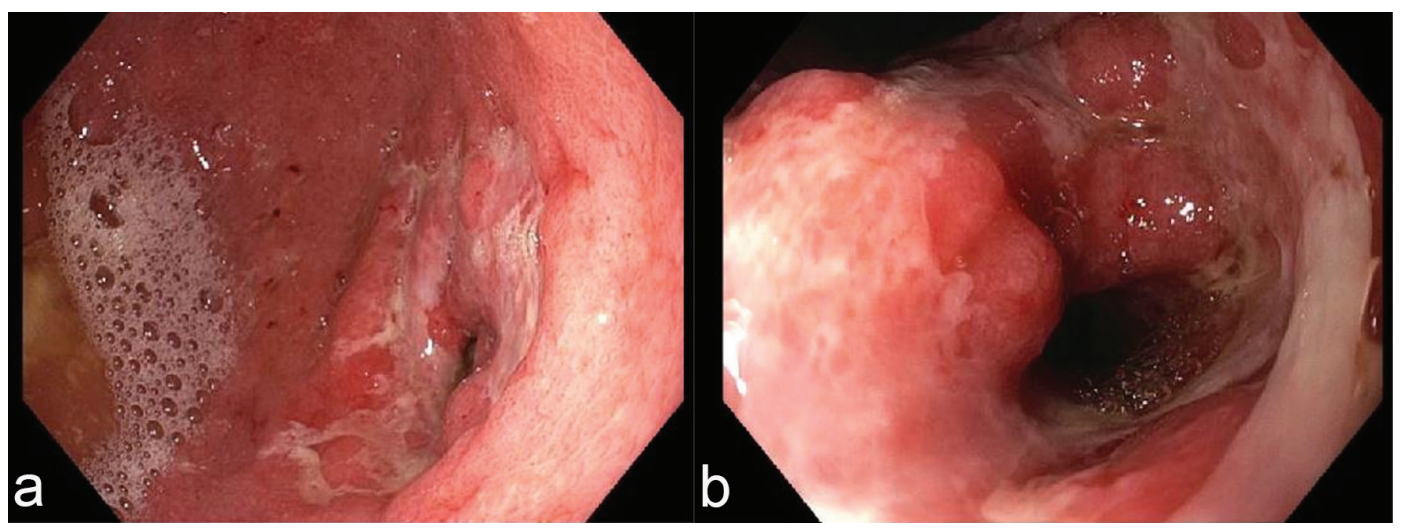

Figure 1. Endoscopic features of the stomach. Esophagogastroduodenoscopy (EGD) revealed diffuse, nodular, erythematous and ulcerated mucosa in the antrum (a) with narrowing of the pyloric channel (b).

Menetrier disease, a disease known as hypoproteinemic hypertrophic gastropathy [12]. Cytomegaloviral gastritis may occur in patients with cancer when they are immunosuppressed as a result of chemotherapy. In cancer patients, there is also a possibility of cytomegaloviral infection in the context of immunotherapy as patients receiving PD-1 inhibitors frequently receiving immunosuppressive therapy to manage immune-related adverse events. The use of immunohistochemistry to rule out this infection is generally advisable in this population with gastritis. However, of the six gastric biopsies from patients with PD-1 blocking therapy-related gastrointestinal adverse events, three were immunostained for cytomegaloviral antigen and all of them were negative [9]. Thus, to our knowledge, PD-1 blockade-associated gastritis with concurrent cytomegaloviral gastritis has not been previously reported. Herein, we reported a case of chronic active gastritis with cytomegaloviral infection in one patient treated with anti-PD L1 and anti-PD 1 immunotherapy.

\section{Case Report}

We presented a 66-year-old woman with a past medical history significant for colon cancer (status post-hemicolectomy in 2006, followed by two rounds of adjuvant chemotherapy) and bladder cancer (status post-neoadjuvant chemotherapy with gemzar and cisplatin). She underwent cystectomy with urostomy. She has metastatic disease to multiple bones, pelvic and para-aortic lymph nodes as revealed on PET scan in November 2016. The patient was treated with atezolizumab for 6 months (beginning on December 21, 2016 to the end of June 2017; 2,000 mg/cycle for a total of eight cycles). Atezolizumab was stopped because of tumor progression and the patient was switched to pembrolizumab (200 mg/cycle) on June 28, 2017 with a total of five cycles prior to the current admission. The patient presented to our hospital with dysphagia, abdominal pain, nausea and intractable vomiting of a few days' duration. Systemic review revealed a 20-pound weight loss. She had no rash, fever, chills, malaise/fatigue, cough, shortness of breath, diarrhea, dysuria, joint pain, myalgia or jaundice. The patient has been on acetaminophen $(325 \mathrm{mg}$ every $6 \mathrm{~h}$ as needed for pain), amoxicillin (2 g/day), bisacodyl (10 mg/day), dronabinol (5 mg/day), lisinopril (10 mg/day) and pantoprazole (40 $\mathrm{mg}$ /day). Physical examination revealed normal body temperature $\left(36.9{ }^{\circ} \mathrm{C}\right)$, pulse rate $(85$ beats $/ \mathrm{min})$, blood pressure $(112 / 72 \mathrm{~mm} \mathrm{Hg})$, respiration rate (16 breaths/min) and body mass index $\left(23.88 \mathrm{~kg} / \mathrm{m}^{2}\right)$. The patient was in no distress. There was tenderness in the epigastric area but without rigidity or guarding. She exhibited edema with trace pitting in the lower legs to the mid tibia. Her urostomy presented with clear yellow urine. Otherwise, her physical examination was unremarkable. There was no evidence of lymphadenopathy, hepatomegaly or splenomegaly. Laboratory tests revealed normal electrolytes, normal liver enzymes (alanine aminotransferase (ALT) $18 \mathrm{IU} / \mathrm{L}$, aspartate aminotransferase (AST) $21 \mathrm{IU} / \mathrm{L}$ and alkaline phosphatase $64 \mathrm{IU} / \mathrm{L})$, slightly elevated total bilirubin at $1.3 \mathrm{mg} / \mathrm{dL}$, slightly low albumin at $3.2 \mathrm{~g} / \mathrm{dL}$, low total protein at $5.6 \mathrm{~g} / \mathrm{dL}$, normal blood urine nitrogen (BUN) $(8 \mathrm{mg} /$ $\mathrm{dL})$, creatinine $(0.98 \mathrm{mg} / \mathrm{dL})$, normal hemoglobin $(12.2 \mathrm{mg} /$ $\mathrm{dL}$ ), white cell counts of $3.7 \times 10^{3} / \mu \mathrm{L}$ and platelet of $111 \times$ $10^{3} / \mu \mathrm{L}$. Abdominal computed tomography revealed gastritis.

Esophagogastroduodenoscopy (EGD) revealed diffuse severely erythematous mucosa with bleeding on contact involving the entire stomach. In addition, diffuse nodular, inflamed and ulcerated mucosa was found in the gastric antrum (Fig. 1a) with narrowing of the pyloric channel (Fig. 1b). This finding led to a clinical suspicion of malignancy. EGD revealed normal duodenum and esophagus. Multiple biopsies were taken from the stomach for histology.

The biopsy from the gastric antrum and body showed similar features which are characterized by marked mononuclear inflammatory cell infiltration in the lamina propria, crypt apoptosis, apoptotic abscesses, crypt dropout, crypt epithelial lymphocytosis and neutrophilic infiltration in crypt epithelium (Fig. 2a-e). Focal erosion and ulceration are noted (Fig. 2f). In addition, a few prominent lymphoid aggregates are noted in the lamina propria. There was no atypia for intraepithelial and lamina propria lymphocytes. A few cytomegalovirus-infected cells were identified on routine stain and further confirmed by immunohistochemistry (Fig. 2g, h). Oxyntic glands in the body mucosa were significantly lost, but no intestinal metaplasia was noted (pictures not shown). The residual glands in the an- 


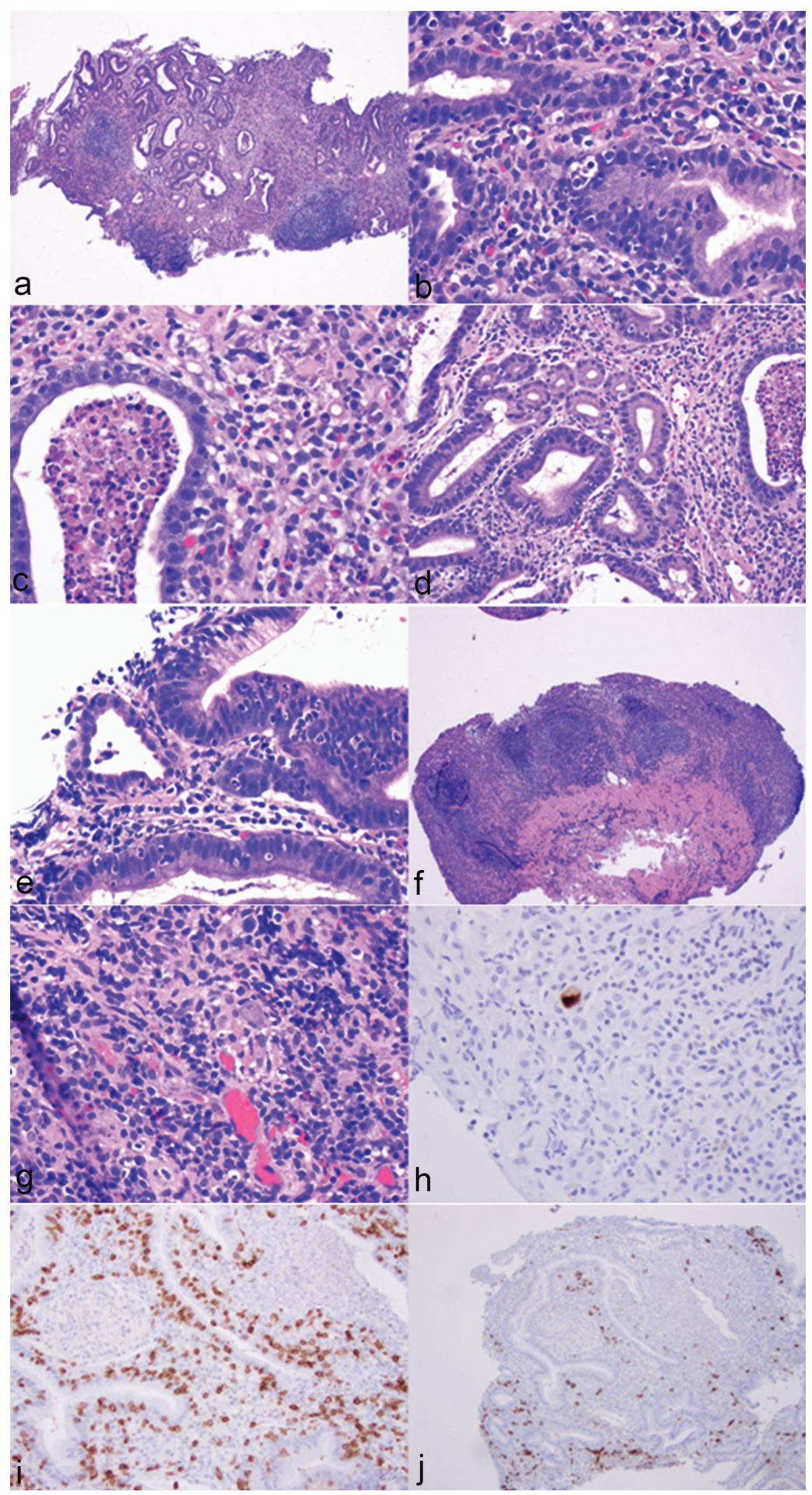

Figure 2. Histological features of immunotherapy-associated gastritis and cytomegaloviral (CMV) infection. The antral mucosa showed marked mononuclear inflammatory cell infiltration in the lamina propria (a, hematoxylin \& eosin stain (H\&E), $\times 40)$, crypt apoptosis (b, H\&E stain, $\times 400$ ), apoptotic abscesses (c, H\&E stain, $\times 400$ ), crypt epithelial lymphocytosis (d, H\&E stain, $\times 200)$ and neutrophilic infiltration in crypt epithelium (e, H\&E stain, $\times 400)$. Focal erosion and ulceration were noted (f, H\&E stain, $\times 40)$. In addition, a few prominent lymphoid aggregates were noted in the lamina propria (f, H\&E stain, $\times 40$ ). The glandular epithelium showed regenerative changes (a). No atypia was noted for lymphocytes (d, f). A few cytomegalovirus-infected cells were noted on routine stain ( $\mathrm{g}, \mathrm{H} \& \mathrm{E}$ stain, $\times 400$ ) and confirmed by immunohistochemistry (h, immunoperoxidase stain, $\times 400$ ). Immunohistochemistry for CD3 and CD20 revealed a mixed population of T cells and B cells with T cells primarily in the crypt epithelium and $B$ cells in the lamina propria (i, immunoperoxidase stain, $\times 200$; j, immunoperoxidase stain, $\times 100$ ). 
trum and body showed regenerative and hyperplastic changes without evidence of dysplasia or carcinoma. No Helicobacter pylori organisms were identified on immunohistochemistry. Immunohistochemistry for CD3 and CD20 revealed a mixed population of $\mathrm{T}$ cells and $\mathrm{B}$ cells with $\mathrm{T}$ cells primarily on the crypt epithelium and B cells in the lamina propria (Fig. 2i, j) and lymphoid follicles (pictures not shown). There was no evidence of lymphoma.

Blood cytomegalovirus (CMV) DNA polymerase chain reaction (PCR) testing ordered after the gastric biopsy revealed CMV DNA was detected, but the load was less than 100 cop$\mathrm{ies} / \mathrm{mL}$ (reference range: not detected). The patient was treated with intravenous ganciclovir $2.5 \mathrm{mg} / \mathrm{kg}$ twice a day for 2 weeks with a plan to switch to valganciclovir for an additional 4 weeks' treatment. Pembrolizumab was held. Five days after intravenous ganciclovir treatment, the patient's symptoms (abdominal pain and vomiting) were significantly improved and she was discharged from the hospital in stable conditions. No corticosteroids were administered to treat her gastritis.

\section{Discussion}

PD-1 and its ligand (PD-L1) blocking agents are novel immunotherapies used for treatment of advanced-stage malignancies. Immune-mediated adverse events in the gastrointestinal tract including autoimmune enteropathy and/or autoimmune colitis, and inflammatory bowel disease-like colitis have been a known phenomenon for these agents [7-9]. The interval between the initiation of anti-PD-1 therapy and the onset of diarrhea is variable and ranges from 1 week to 19 weeks with a median interval of 3 months $[2,13]$. The most common symptom is diarrhea $(89 \%)$ which was sometimes severe and/or bloody. This inflammatory process usually involves multiple sites of the gastrointestinal tract. Endoscopically, the terminal ileum is ulcerated in $40 \%$ of the patients. The colonic mucosa is normal in $35 \%$ of the patients. There is mild colitis in $18 \%$, and marked changes including erosion and friability, either diffuse or patchy, in $47 \%$ of the patients [9]. There were also endoscopic changes in the duodenum and stomach in $67 \%$ and $50 \%$ of the patients [9]. In these cases, gastric involvement is often part of the gastrointestinal tract manifestation. Isolated PD-1 blockage-associated gastritis is rare. In one series of 20 patients, one case $(5 \%)$ presented with only nausea, vomiting, volume depletion and fatigue and was found to have congested, erythematous and granular gastric mucosa on endoscopy [9].

Our patient developed abdominal pain, nausea and vomiting, and weight loss after receiving initial anti-PD-L1 therapy and subsequent anti-PD-1 treatment. Our patient did not have diarrhea indicating that intestine was not affected. EGD in our patient only revealed stomach abnormalities; her duodenum or esophagus were normal endoscopically. The biopsy from the stomach revealed marked destructive gastritis corresponding to the endoscopic finding. The histological features in our case are similar to the patterns previously reported [9] and include lamina propria expansion by mononuclear inflammation, epithelial injury from apoptosis, apoptotic cryptitis, to crypt dropout, and intraepithelial lymphocytic and neutrophilic in- flammation. Thus, the gastritis in this case was felt to be related to PD-1/PD-L1 blockade, at least partially. However, our patient received anti-PD-L1 and anti-PD-1 treatment prior to her presentation; thus, it is impossible to determine which drug is responsible for the development of her gastritis. Given the 2-month interval between cessation of atezolizumab and the symptoms, pembrolizumab may likely to be the culprit; however, a possible role of atezolizumab in the development of gastritis cannot be completely ruled out. Other potential differential diagnoses for the gastritis in our patient include $H$. pylori infection, pre-existing autoimmune gastritis and other medication(s)-related gastritis. The lack of $H$. pylori organism by immunohistochemistry helped rule out $H$. pylori gastritis. Lack of intestinal metaplasia and involvement of antrum helped exclude pre-existing autoimmune gastritis. While the patient has been on multiple medications, none of the medications were known to cause this pattern of severe gastritis.

Given the presence of crypt apoptosis, cytomegaloviral infection should always be considered. Cytomegaloviral gastritis may occur in patients with cancer when they are immunosuppressed as a result of chemotherapy. In cancer patients, there is also a possibility of cytomegaloviral infection in the context of immunotherapy as patients receiving PD-1 inhibitors frequently receiving immunosuppressive therapy to manage immunerelated adverse events. Although eight cases of gastrointestinal biopsies including three gastric biopsies were stained for cytomegalovirus by immunohistochemistry and none of them were positive $[9,14]$, our case had cytomegaloviral infection in the stomach which was demonstrated by histology and immunohistochemistry. CMV gastritis in our patient was further supported by the positive blood CMV DNA result (although at a lower titer of less than 100 copies $/ \mathrm{mL}$ ). Although cytomegaloviral gastritis with prominent lymphoid tissue was reported previously [11], our current case has exuberant chronic inflammation with expansion of the lamina propria and prominent intraepithelial lymphocytosis, features felt to be a more immune-mediated process. Given the use of anti-PD-L1 and anti-PD-1 immunotherapy for 9 months, these two features would be in line with immunotherapy-related gastritis. The florid mononuclear inflammatory infiltrate and lymphoid hyperplasia may explain the endoscopic impression of gastric mucosa nodularity and pyloric channel narrowing. Our patient presented with nausea, vomiting and weight loss, and did have hypoproteinemia, hypoalbuminemia and slight edema in her lower extremities. Endoscopy revealed nodular mucosa and histology revealed marked hyperplasia of the foveolar epithelium and mucus glands and loss of oxyntic glands. These features are suggestive of a dwarf form or a localized form of Menetrier disease.

Treatment for immune-related adverse effects in patients receiving PD-1 blocking agents varies. Patients with grade 2 and grade 3 adverse effects are usually managed by withdrawal of the drug and initiation of corticosteroids [9, 14]. In rare cases, infliximab may be added to treat the patients with severe or persistent symptoms [9, 14]. Gastrointestinal symptoms improved or resolved in $89-100 \%$ of patients after the above mentioned management/treatment $[9,14]$, but few patients had persistent diarrhea despite steroid therapy [9]. Interestingly, in a minority of cases, symptoms/colitis can recur a few months 
after cessation of anti-PD-1 therapy [14]. The treatment in our patient was challenging as there were two drugs (anti-PD-L1 and anti-PD-1) potentially involved and the concurrent cytomegaloviral infection. After the diagnosis of PD-1 blockadeassociated gastritis and cytomegaloviral gastritis, our patient had stopped pembrolizumab temporarily and was treated with ganciclovir. At 5 days after intravenous ganciclovir treatment, the patient's symptoms were significantly improved.

Our case report has several limitations. First, the sequential use of anti-PD-L1 and anti-PD-1 did not allow us to tell which medication was responsible for the development of severe gastritis. In addition, the relationship between immunotherapy-associated immune dysregulation and cytomegaloviral infection was not clear: were they coincidental findings that just happened to occur at the same time, was there an exaggerated immune response to cytomegaloviral infection because of PD-1 blockade, or was cytomegaloviral infection superimposed on immunotherapy-associated gastritis? Third, the clinical follow-up of this case is short and there is neither endoscopic nor histological follow-up. Nonetheless, we report the first case of concurrent immunotherapy-associated and cytomegaloviral gastritis to highlight the need to consider cytomegalovirus and other opportunistic infections in gastrointestinal biopsies from patients receiving PD-1 blocking agents.

\section{Grant Support}

None.

\section{Disclosure}

None.

\section{References}

1. Buchbinder EI, Desai A. CTLA-4 and PD-1 pathways: similarities, differences, and implications of their inhibition. Am J Clin Oncol. 2016;39(1):98-106.

2. Hofmann L, Forschner A, Loquai C, Goldinger SM, Zimmer L, Ugurel S, Schmidgen MI, et al. Cutaneous, gastrointestinal, hepatic, endocrine, and renal side-effects of anti-PD-1 therapy. Eur J Cancer. 2016;60:190-209.

3. Larkin J, Chiarion-Sileni V, Gonzalez R, Grob JJ, Cowey CL, Lao CD, Schadendorf D, et al. Combined nivolumab and ipilimumab or monotherapy in untreated melanoma. N Engl J Med. 2015;373(1):23-34.

4. Topalian SL, Hodi FS, Brahmer JR, Gettinger SN, Smith DC, McDermott DF, Powderly JD, et al. Safety, activity, and immune correlates of anti-PD-1 antibody in cancer. N Engl J Med. 2012;366(26):2443-2454.

5. Robert C, Long GV, Brady B, Dutriaux C, Maio M, Mortier L, Hassel JC, et al. Nivolumab in previously untreated melanoma without BRAF mutation. N Engl J Med. 2015;372(4):320-330.

6. Brahmer J, Reckamp KL, Baas P, Crino L, Eberhardt WE, Poddubskaya E, Antonia S, et al. Nivolumab versus docetaxel in advanced squamous-cell non-small-cell lung cancer. N Engl J Med. 2015;373(2):123-135.

7. McDermott DF, Sosman JA, Sznol M, Massard C, Gordon MS, Hamid O, Powderly JD, et al. Atezolizumab, an anti-programmed death-ligand 1 antibody, in metastatic renal cell carcinoma: long-term safety, clinical activity, and immune correlates from a phase IA study. J Clin Oncol. 2016;34(8):833-842.

8. Balar AV, Galsky MD, Rosenberg JE, Powles T, Petrylak DP, Bellmunt J, Loriot Y, et al. Atezolizumab as first-line treatment in cisplatin-ineligible patients with locally advanced and metastatic urothelial carcinoma: a single-arm, multicentre, phase 2 trial. Lancet. 2017;389(10064):6776.

9. Gonzalez RS, Salaria SN, Bohannon CD, Huber AR, Feely MM, Shi C. PD-1 inhibitor gastroenterocolitis: case series and appraisal of 'immunomodulatory gastroenterocolitis'. Histopathology. 2017;70(4):558-567.

10. Ruiz AR, Jr., Borum ML. Cytomegalovirus hemorrhagic gastritis. AIDS Patient Care STDS. 2001;15(1):1-5.

11. Zucker GM, Otis C, Korowski K, Navab F. Cytomegaloviral gastritis associated with pseudolymphoma. J Clin Gastroenterol. 1994;18:222-226.

12. Sferra TJ, Pawel BR, Qualman SJ, Li BU. Menetrier disease of childhood: role of cytomegalovirus and transforming growth factor alpha. J Pediatr. 1996;128(2):213219.

13. Baroudjian B, Lourenco N, Pages C, Chami I, Maillet M, Bertheau P, Bagot M, et al. Anti-PD1-induced collagenous colitis in a melanoma patient. Melanoma Res. 2016;26(3):308-311.

14. Chen JH, Pezhouh MK, Lauwers GY, Masia R. Histopathologic features of colitis due to immunotherapy with Anti-PD-1 antibodies. Am J Surg Pathol. 2017;41(5):643654. 